\title{
High speed spectroscopy of intermediate polars
}

\section{S. Bloemen*}

Instituut voor Sterrenkunde, Katholieke Universiteit Leuven, Celestijnenlaan 200 D, B-3001

Leuven, Belgium

E-mail: steven.bloemen@ster.kuleuven.be

\section{Steeghs}

Department of Physics, University of Warwick, Coventry CV4 7AL, UK

E-mail: d.t.h.steeghs@warwick.ac.uk

\section{T. R. Marsh}

Department of Physics, University of Warwick, Coventry CV4 7AL, UK

E-mail: t.r.marsh@warwick.ac.uk

To study the accretion process in intermediate polars, spin resolved spectroscopy is an unrivaled tool. Unfortunately, obtaining spin resolved spectroscopy of the intermediate polars with the fastest spinning white dwarfs is challenging. We obtained high speed spectroscopic observations of DQ Her and V455 And. For the latter, which is two magnitudes fainter than DQ Her, we used the QUCAM2 electron-multiplier CCD (EMCCD) installed on the ISIS spectrograph at the William Herschel Telescope. Our analysis of the QUCAM2 data (which will be presented elsewhere) demonstrates that EMCCDs make it possible to observe relatively faint targets at high cadence without burying the signal under the readout noise.

High Time Resolution Astrophysics IV - The Era of Extremely Large Telescopes - HTRA-IV,

May 5-7, 2010

Agios Nikolaos, Crete, Greece

\footnotetext{
${ }^{*}$ Speaker.
} 
Intermediate polars (IPs) are cataclysmic variables containing a moderately magnetic white dwarf. In the inner regions surrounding the accreting white dwarf (WD), the accretion of material from the accretion disc onto the WD is believed to be magnetically controlled. Light curves of IPs typically show variability on the orbital period, the WD spin period and often at various beat periods. The variability on the spin period was thought to arise from disc reprocessing of X-rays that are emitted from the WD's magnetic poles due to energy release of the in-falling material (see e.g. the review on IPs [1]). A more recent suggestion is that the matter gets accreted onto the WD via accretion curtains, and that the spin phase dependent orientation of the curtains gives rise to the observed variability.

Studying the variations of spectral lines on the dwarf spin period, promises to give more insight in the accretion geometry, because it allows us to trace the dynamics of the emitting regions as a function of the spin phase and compare the radial velocity behaviour with proposed accretion geometries. For some IPs with slowly spinning white dwarfs, which have spin periods of tens of minutes, spin resolved spectroscopy has been presented already (see e.g. [2] and [3]).

Spin resolved spectroscopy of IPs with short WD spin periods is much more challenging because of the need for short integration times. Recently, Bloemen et al. (2010) [4] presented spin resolved spectroscopy of the prototype of the intermediate polars, DQ Her, which has a WD spin period of 71 or $142 \mathrm{~s}$ [4] and a magnitude $V \sim 14.5$ [5]. Fig. 1 is taken from [4] and shows the region of the DQ Her spectra around He II $\lambda 4686$. The spectra have been phasefolded on the spin phase into 20 bins and the average spectrum has been subtracted, such that the deviations from the average spectrum are visible. The black and white regions indicate higher and lower than average fluxes respectively. Variations can clearly be seen in the redshifted part of the He II $\lambda 4686$ emission line, but not in the blueshifted part. A simple reprocessing of X-rays from one of the WD's magnetic poles in the accretion disc would lead to emission at the location of the black sine curve on the plot, and can thus not explain the observed variations. Possibly, an accretion curtain geometry can account for the observed effects, but detailed modelling is required to check this possibility. (See [4] for a more elaborate discussion and spin trails of other lines.)

To check whether the DQ Her line variations on the spin period are the rule or rather an exception among IPs with rapidly spinning WDs, we obtained spin resolved spectroscopy of V455 And, which has a spin period of $67.6 \mathrm{~s}$ and is about two magnitudes fainter than DQ Her [6]. To resolve the WD spin phases, the integration times of the spectra are limited to a few seconds. Using a 4-m class telescope, the spectra would be dominated by read-out noise if we would have used a standard CCD. Instead, we made use of the QUCAM2 electron-multiplier CCD (EMCCD) installed on the ISIS spectrograph at the 4.2-m William Herschel Telescope at La Palma to obtain 15796 spectra with exposure times of $2 \mathrm{~s}$. The results of our analysis clearly demonstrate the power of EMCCDs for high speed spectroscopy of relatively faint targets and will be discussed in [7].

\section{Acknowledgments}

The observations were made with the William Herschel Telescope operated at the island of La Palma by the Isaac Newton Group in the Spanish Observatorio del Roque de los Muchachos of the Instituto de Astrofísica de Canarias.

The research leading to these results has received funding from the European Research Council under the European Community's Seventh Framework Programme (FP7/2007-2013)/ERC grant 


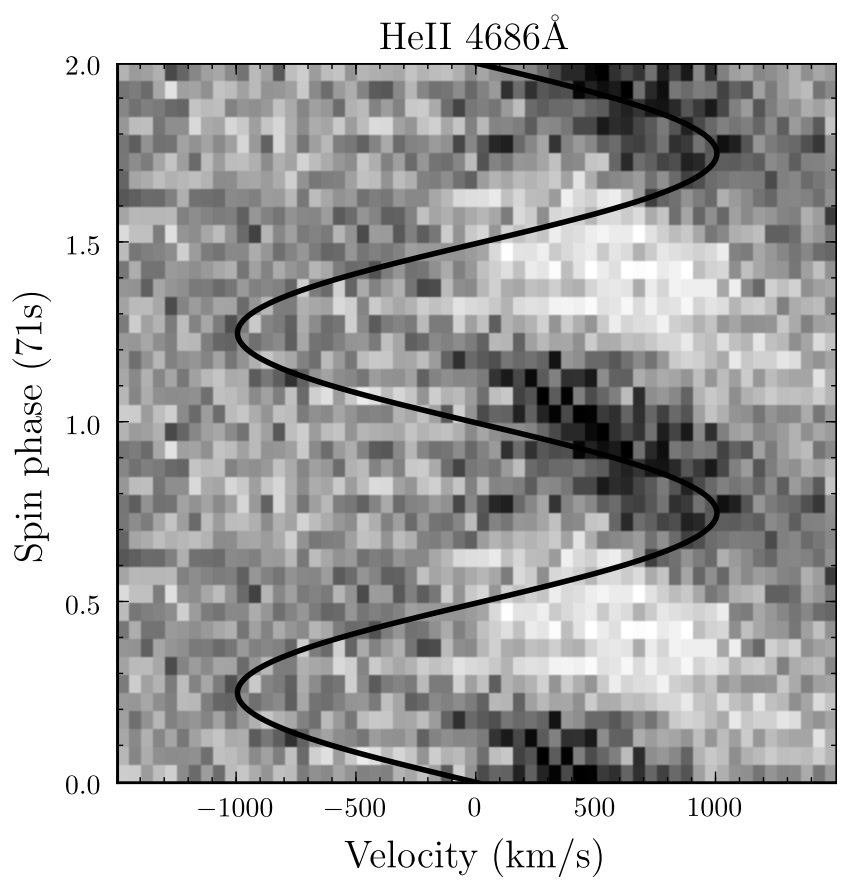

Figure 1: Spintrail of the variations in DQ Her's He II $\lambda 4686$ line profile, created by phasefolding the spectra and subtracting the average spectrum. Enhanced emission (darker region) is seen once per spin phase in the red-shifted part of the line, but not in the blue-shifted part. The black sinusoidal curve is a simple model for the expected enhanced emission if the emission would be reprocessed light of one white dwarf X-ray beam in a small part of the accretion disc. (Figure taken from [4]. See the original paper for more details.)

agreement $\mathrm{n}^{\circ} 227224$ (PROSPERITY), as well as from the Research Council of K.U.Leuven grant agreement GOA/2008/04. During this research DS and TRM were supported under grants from the UK's Science and Technology Facilities Council (STFC, ST/F002599/1 and PP/D005914/1).

\section{References}

[1] J. Patterson, The DQ Herculis stars, 1994, PASP, 106, 209

[2] C. Hellier, M. Cropper, \& K. O. Mason, Optical and X-ray observations of AO PISCIUM and the origin of the spin pulse in intermediate polars, 1991, MNRAS, 248, 233

[3] C. Hellier, Spectroscopy of the intermediate polars BG Canis Minoris and PQ Geminorum, 1997, MNRAS, 288, 817

[4] S. Bloemen, T. R. Marsh, D. Steeghs, \& R. H. Østensen, Spin-resolved spectroscopy of the intermediate polar DQ Her, 2010, MNRAS, 970

[5] E. Zhang, E. L. Robinson, R. F. Stiening, \& K. Horne, The 71 Second Oscillation in the Light Curve of the Old Nova DQ Herculis, 1995, ApJ, 454, 447

[6] Araujo-Betancor, S., et al., HS 2331+3905: The cataclysmic variable that has it all, 2005, A\&A, 430, 629 
[7] S. Bloemen, D. Steeghs, K. De Smedt, J. Vos, B. T. Gänsicke, T. R. Marsh, \& P. Rodriguez-Gil, Spin-resolved spectroscopy of the intermediate polar V455 And using an electron-multiplying CCD, in preparation 